Research Article

\title{
Thinned Virtual Array for Cramer Rao Bound Optimization in MIMO Radar
}

\author{
Xia Li iD and Buhong Wang (iD \\ Information and Navigation College, Air Force Engineering University, Xi'an 710077, China \\ Correspondence should be addressed to Buhong Wang; wbhgroup@aliyun.com
}

Received 31 July 2020; Revised 5 December 2020; Accepted 28 December 2020; Published 15 January 2021

Academic Editor: Qing Shen

Copyright (๑) $2021 \mathrm{Xia} \mathrm{Li} \mathrm{and} \mathrm{Buhong} \mathrm{Wang.} \mathrm{This} \mathrm{is} \mathrm{an} \mathrm{open} \mathrm{access} \mathrm{article} \mathrm{distributed} \mathrm{under} \mathrm{the} \mathrm{Creative} \mathrm{Commons} \mathrm{Attribution}$ License, which permits unrestricted use, distribution, and reproduction in any medium, provided the original work is properly cited.

\begin{abstract}
By transmitting multiple independent waveforms at the transmit side and processing echoes of spatial targets at the receive side, Multiple Input Multiple Output (MIMO) radar enjoys virtual array aperture expansion and more degree of freedom (DOF), both of which favors the application of direction finding or estimation of direction of arrival (DOA). The expanded virtual aperture provides higher angular resolution which also promotes the precision of DOA estimation, and the extra DOF brought by waveform diversity can be leveraged to focus energy in certain spatial region for better direction-finding capacity. However, beamspace methods which match certain beampatterns suffer from deteriorated performance and complexity in implementation, and the advantage of virtual array aperture is limited by its virtual element redundancy. As an important performance indicator of DOA estimation, Cramer-Rao Bound (CRB) is closely connected to the array configuration of the system. To reduce the complexity of the system and improve CRB performance at the same time, in this paper, the virtual array of MIMO radar is designed directly by selecting outputs from matched filters at the receive side. For the sake of fair comparison, both scenarios with and without priori directions are considered to obtain optimized virtual array configuration, respectively. The original combinatorial problems are approximated by sequential convex approximations methods which produce solutions with efficiency. Numerical results demonstrate that the proposed method can provide thinned virtual arrays with excellent CRB performance.
\end{abstract}

\section{Introduction}

In various applications of radar system, such as beamforming and interference suppression, the Direction of Arrival (DOA) is often needed as a priori information [1]. Inversely, the performance of DOA estimation can be improved by proper beamforming and interference suppression [2]. Therefore, DOA estimation is a prerequisite for various applications as well as the purpose after other processing procedures, which is of great importance to the array of the radar system [3].

The performance of DOA estimation is related to the properties of array, which includes the aperture and structure of the array. In MIMO radar, each element of transmit array sends one of multiple independent waveforms, the echoes of which are received by the receive array and processed through matched filters. The data after matched filtering operation is equivalent to the one received by an array with larger array aperture, which is often referred to as virtual array. The aperture of virtual array is usually larger than that of both transmit and receive array, which is helpful for DOA estimation since larger aperture is productive for angle resolution [4]. However, such expansion of virtual array aperture is limited by the redundancy of virtual array elements rendered by the adoption of uniform array configuration at both transmit and receive side. Even though both transmit array and receive array are uniformly distributed without spatial tapering in most cases of MIMO radar, the virtual array configuration obtained by the spatial convolution of transmit array and receive array is characterized by the fact that one element in the virtual array may correspond to several pairs consisting of elements from both transmit array and receive array, producing redundancies of virtual array elements [5]. Such redundancy not only leads to the waste of element 
resources and increase of hardware complexity but also produces array shading effect which often adds unwanted weights on the virtual array elements, contributing to the reduction of array performance. To tackle the redundancy of virtual array and reduce the hardware cost and complexity of the system, references [6-8] propose to model the virtual array as polynomial and decompose the polynomial to obtain thinned polynomials which represent both transmit array and receive array. In these problems, the virtual array is given as a uniform one to eliminate negative effects brought by unwanted weights in the virtual array and obtain thinned transmit and receive arrays to reduce hardware complexity. However, the number of possible solutions, i.e., the possible combinations of thinned transmit and receive arrays, are limited due to the intrinsic nature of spatial convolution. To enlarge the scope of solution space and obtain larger virtual aperture while preserving the thinned transmit array and receive array, mathematical tools such as difference sets [9], cyclic difference sets [10], and almost difference sets [11], as well as numerical optimization methods such as genetic algorithm [12] and simulated annealing [13] are proposed to obtain more available solutions for thinned transmit and receive array given the same number of elements, whereas the optimality of solutions depends on the initial points for the numerical problems and the computational complexity of the problem is much larger than polynomial decomposition. To obtain an even larger virtual aperture with thinned transmit array and receive array, coprime array [14-16] and nested array [17-19] are proposed together with the difference coarray processing method, which utilizes $O(N)$ elements to achieve $O\left(N^{2}\right)$ or even $O\left(N^{4}\right)$ DOF. In this way, larger DOF promotes parameter identifiability and angular resolution of the array, but such difference co-array-based array configuration is prone to error and distortion from the multipath effect in practical applications. These array configurations for the MIMO radar are focused on improving DOA estimation performance by expanding virtual array aperture using thinned transmit array and receive array [20]. However, larger virtual aperture does not necessarily translate to higher estimation precision of the MIMO radar [21].

The performance of DOA estimation depends on the effective array aperture on the direction to be estimated, which means that the array configuration with the best DOA estimation performance is determined by the DOA to be estimated [22]. In fact, as a metric representing the limit performance of array and DOA estimation, the Cramer-Rao Bound (CRB) is closely related to the array configuration of the system and the estimated direction [23]. Meanwhile, in practical applications of direction finding, priori information on the estimated direction is often available. Therefore, in order to fully utilize DOF from the configuration of the virtual array to achieve lower total CRB, a thinned virtual array is proposed. The work of this paper is summarized as follows:

(1) The signal model of the MIMO radar is proposed, in which the virtual array expansion and the connection between virtual array configurations are discussed. Since the performance of DOA estimation or
CRB of the MIMO radar depends on the structure of virtual array, the connection between virtual array structure, fisher information matrix (FIM), and CRB is discussed to reveal the impact of array configuration on the direction-finding performance of certain spatial targets, and analytical expressions are derived.

(2) Due to the uniformity in the distribution of elements in both transmit array and receive array, elements in the virtual array are redundant. Reducing such redundancy not only decreases the hardware complexity and waste of system resources but also provides potential for utilizing the DOF provided from the configuration of the array by incorporating priori DOA information in the structure of thinned array to achieve lower total CRB of the system. In this way, the redundancy of the virtual array structure is analysed and relation between the total CRB and virtual array configuration is generalized to derive the CRB expression of thinned virtual array configuration.

(3) Based on these analyses, the optimization problem for obtaining thinned virtual array is proposed. Both the scenario with and without directional information are considered. Because of the fractional structure and nonconvex constraints, the solution to the original form of the proposed problem requires enumeration with high-computational complexity. To solve the problem efficiently, the Dinkelbach method is used to approximate the original problem into a series of subproblems, all of which can be solved iteratively. The final solution to the problem is obtained by solving another optimal integer constraint problem. Numerical results show CRB and DOA estimation performance to validate the advantage of the proposed thinned virtual array.

It should be noted that the goal of this paper is thinning the virtual array configuration directly through turning off some of matched filters on the receive side rather than designing thinned transmit and receive arrays to obtain thinned virtual array. Compared to thinning transmit array and receive array, respectively, the proposed scheme has the following advantages:

(1) Even though thinning transmit and receive arrays separately achieve more reduction of hardware resources than thinning virtual array directly, such separated scheme has fewer DOF for increased performance due to limited number of virtual array structures formed by spatial convolution of transmit and receive array structures, whose combinations are also limited. Meanwhile, the joint scheme can produce arbitrary virtual array configuration by turning off matched filters and thus enjoys more possibilities for increased system performance.

(2) The direct thinning of virtual array entails a precondition that the virtual array must be fully filled or at least one virtual element exists at the sensor 
positions of the obtained array. In other words, the joint scheme requires both transmit array and receive array to be fully filled, which appears to waste hardware resources than that of the separated scheme. However, the precondition of fully filled also implies that the proposed joint scheme can be achieved without changing configuration of physical elements in transmit array and receive array. This enables fast deployment of the proposed scheme on obsolete systems with filled arrays, and fast reconfiguration of virtual array on the MIMO radar at work. The proposed scheme is neither better than any other existing ways of thinning virtual arrays nor the one with lowest cost or fastest speed. Rather, this scheme is proposed to provide a possibility to improve the direction-finding performance with more DOF on existing MIMO radar systems.

The rest of the paper is organized as follows. Section 2 introduces the signal model, which deals with virtual array aperture expansion in the MIMO radar, redundancy in virtual array structure, and the connection between virtual array and CRB. The design of thinned virtual array for optimization of CRB is discussed in Section 3, considering both scenarios with and without priori directional information. Numerical results are provided in Section 4, followed by conclusion in Section 5 .

\section{Signal Model}

Consider a MIMO radar system with transmit array of $M_{t}$ elements and receive array of $M_{r}$ elements. The position of the $m$ th transmit array element is $\left(x_{m}, y_{m}\right)(m=1, \ldots$, $M_{t}$ ) and the position of the $n$th receive array element is $\left(x_{n}, y_{n}\right)\left(n=1, \ldots, M_{r}\right)$. Assume that the distance between transmit and receive arrays is much fewer than wavelength $\lambda$ so that the target direction with respect to transmit array is the same as the target direction with respect to receive array, and all elements in both transmit and receive arrays share the same carrier frequency $F_{c}$. It is also assumed that there are $L$ targets with elevation angles $\theta_{l}(l=1, \ldots, L)$ and azimuth angles $\phi_{l}(l=1, \ldots, L)$. In the transmit array, each element transmits an orthogonal waveform towards the target, and the complex envelope of the waveform at the $m$ th element can be denoted as

$$
s_{m}(t)=\rho \phi_{m}(t), \quad m=1, \ldots, M_{t},
$$

where $t$ denotes fast-time index, i.e., the time index within a radar pulse, $\rho$ is the energy coefficient of waveform to normalize the sum of energy of all transmitted waveforms as constant, And $\phi_{m}(t)$ is the $m$ th orthogonal baseband waveform. After up conversion, the signal sent from the $m$ th element can be correspondingly denoted as $s_{m}(t) \exp$ $\left(j 2 \pi F_{c} t\right)$, where the term $\exp \left(j 2 \pi F_{c} t\right)$ represents the carrier.

The radar echo signal at the $l$ th target is represented as

$$
r_{1}(t, \tau)=\rho \beta_{l}(\tau) \mathbf{a}^{T}\left(\theta_{l}\right) \phi(t) \exp \left(j 2 \pi F_{c} t\right),
$$

where $\beta_{l}(\tau)$ denotes the reflection coefficient of the $l$ th target, $\mathbf{a}\left(\theta_{l}\right)$ is the transmit steering vector corresponding to the $l$ th target, and $\phi(t)=\left[\phi_{1}(t), \phi_{2}(t), \ldots, \phi_{M_{l}}(t)\right]^{T}$ is the vector consists of $M_{t}$ orthogonal waveforms. It should be noted that the reflection coefficient within a single radar pulse remains the same yet different between different pulses.

All orthogonal waveforms from the transmit array is reflected from the target and captured by the receive array. The received signal is then down-converted to baseband by multiplying with $\exp \left(-j 2 \pi F_{c} t\right)$. In this way, the $M_{r} \times 1$ baseband equivalent of received echo signal can be expressed as

$$
\mathbf{x}(t, \tau)=\sum_{l=1}^{L} r_{l}(t, \tau) \exp \left(-j 2 \pi F_{c} t\right) \mathbf{b}\left(\theta_{l}\right)+\mathbf{z}(t, \tau),
$$

where $\tau$ represents slow time index, which is the index of radar pulse, $\mathbf{b}\left(\theta_{l}\right)$ denotes the steering vector corresponding to the $l$ th target with direction $\theta_{l}$, and $\mathbf{z}(t, \tau)$ is an $M_{r} \times 1$ Gaussian white noise.

2.1. Virtual Array Aperture Expansion. The transmit steering vector and receive steering vector can be expressed as

$$
\begin{aligned}
& \mathbf{a}(\theta)=\exp \left[j k_{0}\left(x_{1} u_{x}+y_{1} u_{y}\right), \ldots, j k_{0}\left(x_{M_{t}} u_{x}+y_{M_{t}} u_{y}\right)\right]^{T}, \\
& \mathbf{b}(\theta)=\exp \left[j k_{0}\left(x_{1} u_{x}+y_{1} u_{y}\right), \ldots, j k_{0}\left(x_{M_{r}} u_{x}+y_{M_{r}} u_{y}\right)\right]^{T},
\end{aligned}
$$

where $k_{0}=(2 \pi / \lambda), u_{x}=\cos \theta \cos \phi$, and $u_{y}=\cos \theta \sin \phi$.

Since the transmitted equivalent waveforms are orthogonal or linearly independent from each other, the component corresponding to a single waveform can be extracted using matched filters on the receive array. After going through the down conversion at the receiver side, the $M_{r} \times 1$ baseband equivalent data vector corresponding to the $m$ th orthogonal waveform can be expressed as

$$
\mathbf{x}_{m}(\tau)=\int_{T} \mathbf{x}(t, \tau) \phi_{m}^{*}(t) \mathrm{d} t, \quad m=1, \ldots, M_{t} .
$$

Stacking all data component vectors corresponding to different waveforms in a columnwise manner, an $M_{t} M_{r} \times 1$ virtual data vector can be obtained as

$$
\begin{aligned}
\mathbf{v}(\tau) & =\rho \sum_{l=1}^{L} \beta_{l}(\tau)\left(\mathbf{a}\left(\theta_{l}\right) \otimes \mathbf{b}\left(\theta_{l}\right)\right)+\widetilde{z}(\tau) \\
& =\rho \sum_{l=1}^{L} \beta_{l}(\tau) \mathbf{u}\left(\theta_{l}\right)+\widetilde{z}(\tau),
\end{aligned}
$$

where

$$
\mathbf{u}(\theta)=\mathbf{a}(\theta) \otimes \mathbf{b}(\theta)
$$

denotes $M_{t} M_{r} \times 1$ virtual array steering vector and $\widetilde{z}(\tau)$ is $M_{t} M_{r} \times 1$ noise term with covariance matrix as $\sigma_{z}^{2} \mathbf{I} M_{t} M_{r}$

It can be inferred from (7) that the virtual data vector can be seen as a signal received by an $M_{t} M_{r} \times 1$ array. This is equivalent to the case that spatial signal is received by a virtual array with larger aperture, which is depicted in Figure 1. Usually, an array with larger aperture implies better 


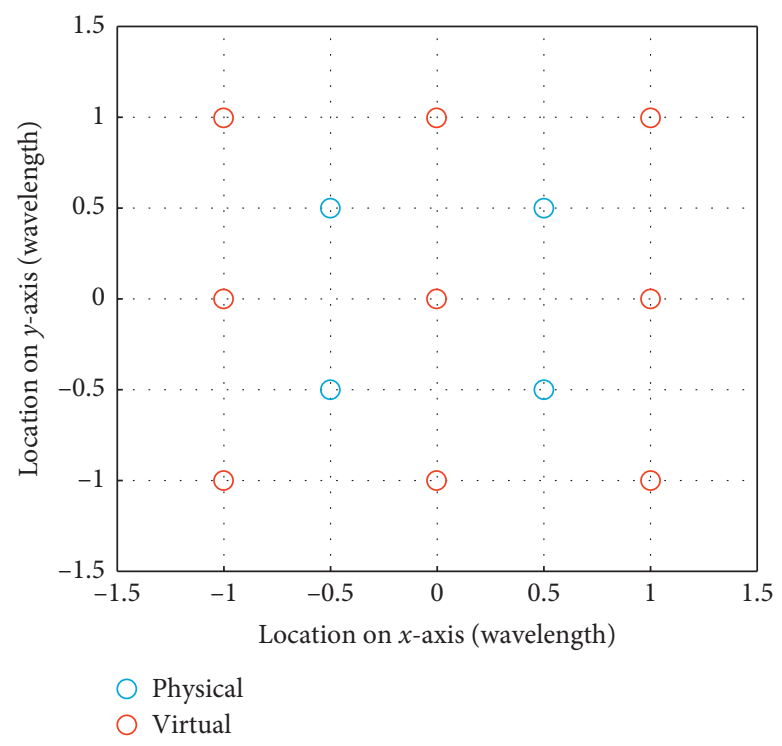

FIGURE 1: Virtual array expansion.

DOA estimation performance, yet this is not the case with MIMO radar virtual array. To demonstrate the effect of virtual array design on the performance of direction finding, the connection between virtual array structure and CRB is to discussed in Section 2.2.
2.2. Connection between Virtual Array and CRB. To simplify subsequent analysis, the following vectors are defined based on the location of array elements:

$$
\begin{aligned}
\mathbf{x} & =\left[x_{1}+x_{1}, x_{1}+x_{2}, \ldots, x_{1}+x_{M_{r}}, x_{2}+x_{1}, \ldots, x_{2}+x_{M_{r}}, \ldots, x_{M_{t}}+x_{M_{r}}\right]^{T}, \\
\mathbf{y} & =\left[y_{1}+y_{1}, y_{1}+y_{2}, \ldots, y_{1}+y_{M_{r}}, y_{2}+y_{1}, \ldots, y_{2}+y_{M_{r}}, \ldots, y_{M_{t}}+y_{M_{r}}\right]^{T}, \\
\mathbf{x}_{\mathbf{x}} & =\left[\left(x_{1}+x_{1}\right)^{2}, \ldots,\left(x_{1}+x_{M_{r}}\right)^{2},\left(x_{2}+x_{1}\right)^{2}, \ldots,\left(x_{2}+x_{M_{r}}\right)^{2}, \ldots,\left(x_{M_{t}}+x_{M_{r}}\right)^{2}\right]^{T}, \\
\mathbf{y}_{\mathbf{y}} & =\left[\left(y_{1}+y_{1}\right)^{2}, \ldots,\left(y_{1}+y_{M_{r}}\right)^{2},\left(y_{2}+y_{1}\right)^{2}, \ldots,\left(y_{2}+y_{M_{r}}\right)^{2}, \ldots,\left(y_{M_{t}}+y_{M_{r}}\right)^{2}\right]^{T}, \\
\mathbf{x}_{\mathbf{y}} & =\left[\left(x_{1}+x_{1}\right)\left(y_{1}+y_{1}\right), \ldots,\left(x_{M_{t}}+x_{M_{r}}\right)\left(y_{M_{t}}+y_{M_{r}}\right)\right]^{T} .
\end{aligned}
$$

Assume that a target locates at $(\theta, \phi)$, where $\theta$ is the elevation and $\phi$ is the azimuth. During one specific pulse, the $k$ th sample signal received by the system is $\mathbf{y}(k), k=$ $0,1, \ldots, N-1$. Stack all data by columns to form new column vector:

$$
\mathbf{z}=\left[\mathbf{v}(0)^{T}, \ldots, \mathbf{v}(N-1)^{T}\right]^{T}
$$

Let the array response of system in the noise-free condition be

$$
\mathbf{u}(k)=\sum_{l=1}^{L} \rho \beta_{l} \mathbf{b}\left(\theta_{l}, \phi_{l}\right) \mathbf{a}^{T}\left(\theta_{l}, \phi_{l}\right) \boldsymbol{\phi}(k)
$$

Then,

$$
\mathbb{E}\{\mathbf{z}\}=\mathbf{u}=\left[\mathbf{u}(0)^{T}, \ldots, \mathbf{u}(N-1)^{T}\right]^{T}
$$

This means that the received data $\mathbf{z}$ conforms to the complex Gaussian distribution with $\mathbf{u}$ as mean and $\sigma_{z}^{2} \mathbf{I}_{M_{r} K}$ as covariance matrix, which also implies that the estimator $\mathbf{Z}$ is the minimum variance unbiased estimator of $\mathbf{u}$. The probability density function (PDF) is

$$
p(\mathbf{z}, \boldsymbol{\Omega})=\frac{1}{\pi^{M_{r} N} \sigma_{z}^{2 M_{r} N}} \exp \left\{-\frac{1}{\sigma_{z}^{2}}(\mathbf{z}-\mathbf{u})^{H}(\mathbf{z}-\mathbf{u})\right\} .
$$

For the existence of minimum variance, it is assumed that the PDF satisfies the regularity condition:

$$
\mathbb{E}\left[\frac{\partial \ln p(\mathbf{z}, \boldsymbol{\Omega})}{\partial \boldsymbol{\Omega}}\right]=0, \quad \text { for all } \boldsymbol{\Omega},
$$

where the expectation operator is taken with respect to the PDF $p(\mathbf{z}, \Omega)$. For each element in the parameter vector $\Omega=[\theta, \phi]^{T}$, the lower bound of the estimation variance of each element exists. Such lower bound is the so-called CRB. 
CRB can be derived by conducting inverse operation on the Fisher information matrix:

$$
\operatorname{var}\left(\widehat{\Omega}_{i}\right) \geq\left[\mathbf{J}^{-1}\right]_{i i}, \quad 1 \leq i \leq 2,
$$

where $\operatorname{var}(\bullet)$ denotes the variance, $\widehat{\Omega}_{i}$ is the estimate of the $i$ th element in the parameter vector, and $\left[\mathbf{J}^{-1}\right]_{i i}$ denotes the $(i, i)$ element in the inverse of the Fisher information matrix $\mathbf{J}$, which is the $i$ th element on the diagonal.

The element of the Fisher information matrix can be given by the following expression:

$$
\mathbf{J}_{i, j}=f\left(\Omega_{i}, \Omega_{j}\right)=\mathbb{E}\left[\frac{\partial \ln p(\mathbf{z}, \mathbf{\Omega})}{\partial \Omega_{i}} \frac{\partial \ln p(\mathbf{z}, \mathbf{\Omega})}{\partial \Omega_{j}}\right] .
$$

The Fisher information matrix, therefore, can be expressed as

$$
\mathbf{J}=\left[\begin{array}{ll}
f(\theta, \theta) & f(\theta, \phi) \\
f(\phi, \theta) & f(\phi, \phi)
\end{array}\right]
$$

Among all elements in the matrix, the Fisher information corresponding to the parameter $\theta$ is

$$
f(\theta, \theta)=G \sin ^{2} \theta\left(Q_{x x} \cos ^{2} \phi+Q_{y y} \sin ^{2} \phi+Q_{x y} \sin (2 \phi)\right),
$$

where $G$ represents the constant term irrelevant to the directions and $Q_{x x}, Q_{y y}$, and $Q_{x y}$ are called inertia momentum, which are defined as follows:

$$
\begin{aligned}
Q_{x x} & =\sum_{m=1}^{M_{t}} \sum_{n=1}^{M_{r}}\left(x_{m}+x_{n}\right)^{2}=\mathbf{1}^{T} \mathbf{X}_{\mathbf{x}}, \\
Q_{y y} & =\sum_{m=1}^{M_{t}} \sum_{n=1}^{M_{r}}\left(y_{m}+y_{n}\right)^{2}=\mathbf{1}^{T} \mathbf{y}_{\mathbf{y}}, \\
Q_{x y} & =\sum_{m=1}^{M_{t}} \sum_{n=1}^{M_{r}}\left(x_{m}+x_{n}\right)\left(y_{m}+y_{n}\right)=\mathbf{1}^{T} \mathbf{x}_{\mathbf{y}} .
\end{aligned}
$$
$\phi$ is

The Fisher information corresponding to the parameter

$$
f(\phi, \phi)=G \cos ^{2} \theta\left(Q_{x x} \sin ^{2} \phi+Q_{y y} \cos ^{2} \phi-Q_{x y} \sin (2 \phi)\right) .
$$

Note that the matrix $\mathbf{J}$ is symmetric, and the cross-term is therefore

$$
\begin{aligned}
f(\theta, \phi) & =f(\theta, \phi) \\
& =\frac{G}{4} \sin (2 \theta)\left[\left(Q_{x x}-Q_{y y}\right) \sin (2 \phi)-2 Q_{x y} \cos (2 \phi)\right] .
\end{aligned}
$$

The derivation of (17), (19), and (20) is provided in Appendix A.

As discussed above, the CRB matrix is defined to be the inverse of the matrix $\mathbf{J}$ so that the CRB of parameter $\theta$ can be expressed as

$$
C(\theta, \theta)=f(\phi, \phi)\left[f(\theta, \theta) f(\phi, \phi)-f^{2}(\theta, \phi)\right]^{-1} .
$$

Similarly, the CRB of parameter $\phi$ can be expressed as

$$
C(\phi, \phi)=f(\theta, \theta)\left[f(\theta, \theta) f(\phi, \phi)-f^{2}(\theta, \phi)\right]^{-1} .
$$

It can be revealed from above that the array configuration has an important impact on CRB through inertia momentum. In the same way, the virtual array configuration has an effect on the CRB of the MIMO radar through inertia momentum of virtual array elements. The redundancy of elements in virtual array from uniform virtual array configuration and the comparison between thinning transmit array and receive array and thinning virtual array are discussed in Section 2.3.

2.3. Redundancy in Virtual Array Structure. It has been mentioned in Section 2.2 that the virtual data vector obtained from the matched filtering on the $M_{r} \times 1$ receive array, which receives echoes of orthogonal waveforms from $M_{t} \times 1$ transmit array, can be considered as the signal received by an $M_{t} M_{r} \times 1$ array. In this way, a virtual array with virtual expanded aperture is achieved, which is helpful for DOA estimation. However, multiple elements occupy the same locations in the virtual array, causing the redundancy in the virtual array. These redundant elements not only renders inadequate use of virtual elements from the transmit array and receive array but also affects the output of virtual elements brought by intrinsic weighting from the redundancy. The redundancy of elements in the virtual array is sketched in Figure 2, where the height on each location denotes the number of repetitive elements in the virtual array aperture, which implies the level of redundancy. Such redundancy cannot be eliminated through the thinning in transmit array or receive array since the virtual array steering vector is constructed by the spatial convolution of transmit and receive steering vectors. Furthermore, the DOF in thinning transmit array and receive array separately is limited due to the fact that once an element in transmit array or receive array is not in their thinned counterparts, all virtual elements corresponding to the discarded element are not in the thinned virtual array. To reduce the redundancy in the virtual array and increase the DOF in the design of thinned virtual array, more flexible ways of thinning are needed.

To serve the purpose of increasing DOF for designing thinned virtual array, the structure of MIMO is considered. In the receive array of the MIMO radar, each element is followed by several matched filters, each of which corresponds to one independent waveforms from transmit array so that each waveform component in the echoes can be separated after match filtering operation. In this way, each element in the receive array is followed by $M_{r}$ matched filters, paired with $M_{t}$ orthogonal waveforms in the transmit array; the total number of matched filters is $M_{t} M_{r}$, which is the same as the dimensions of virtual steering vector and virtual data vector. In fact, thanks to the orthogonality of waveforms, each output data component from each matched filters is equivalent to the output from element in the virtual array which takes virtual location determined by its 


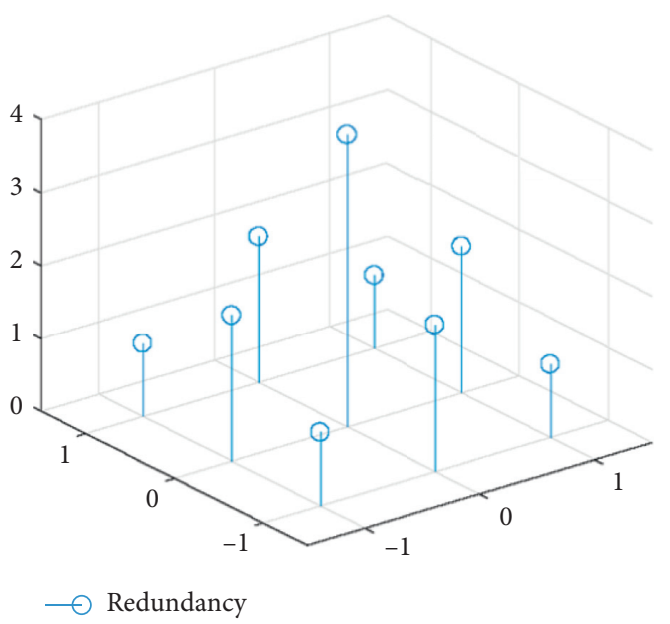

FIGURE 2: Redundancy in virtual array.

$$
\begin{aligned}
& Q_{w x x}=\mathbf{w}^{T} \mathbf{x}_{\mathbf{x}}, \\
& Q_{w y y}=\mathbf{w}^{T} \mathbf{y}_{\mathbf{y}}, \\
& Q_{w x y}=\mathbf{w}^{T} \mathbf{x}_{\mathbf{y}} .
\end{aligned}
$$

In this way, the element in the Fisher information matrix of thinned virtual array can be rewritten as

$$
\begin{aligned}
& f_{w}(\theta, \theta)=G \sin ^{2} \theta \cdot\left(Q_{w x x} \cos ^{2} \phi+Q_{w y y} \sin ^{2} \phi+Q_{w x y} \sin (2 \phi)\right), \\
& f_{w}(\phi, \phi)=G \cos ^{2} \theta \cdot\left(Q_{w x x} \sin ^{2} \phi+Q_{w y y} \cos ^{2} \phi+Q_{w x y} \sin (2 \phi)\right), \\
& f_{w}(\theta, \phi)=\frac{G}{4} \sin (2 \theta) \cdot\left[\left(Q_{w x x}-Q_{w y y}\right) \sin (2 \phi)-2 Q_{w x y} \cos (2 \phi)\right] .
\end{aligned}
$$

\section{Design of Thinned Virtual Array for Optimization of CRB}

In Section 2.2, the connection between virtual array configuration and CRB is revealed, indicating the reduction redundancy and inflexibility in virtual array design can be avoided by directly thinning virtual array through selection of matched filters on the receiver array. The specific configuration of thinned virtual array, however, should be considered carefully in the specific condition. If priori direction of the target is not available or not considered, the thinned virtual array with the best CRB performance is the one that meets the isotropic condition; if priori direction of target is available, the virtual array with optimized CRB performance depends on the given information of the target. Note that the optimization of CRB usually takes the trace minimization of the CRB matrix, namely, to solve the following problem:

$$
\min _{\mathbf{c}} \operatorname{tr}(\mathbf{C}) \text {. }
$$

The thinned virtual array without or with priori direction is to be considered in the following.

3.1. Thinned Virtual Array without Priori Direction. In the case where no priori information on target direction is available, the best thinned virtual array is isotropic, namely, the CRB performance should be decoupled from elevation and azimuth angles of the target. Therefore, the following conditions is to be satisfied:

$$
\begin{aligned}
& Q_{w x x}=Q_{w y y}=Q \\
& Q_{w x y}=0
\end{aligned}
$$

so that the $\mathrm{CRB}$ matrix can be rewritten as

xpressed as 


$$
\mathbf{C}=\mathbf{J}^{-1}=\frac{1}{G}\left[\begin{array}{cc}
\frac{1}{\left(\cos ^{2} \theta\right) Q} & 0 \\
0 & \frac{1}{\left(\sin ^{2} \theta\right) Q}
\end{array}\right] \text {, }
$$

which also implies

$$
\min _{Q} \frac{1}{\left(\sin ^{2} \theta \cos ^{2} \theta\right) Q}
$$

Note that (32) holds for any angle $\theta$. The problem can thus be rewritten as

$$
\max _{Q} Q \text {. }
$$

It can be implied from (25), (26), and (33) that the optimized isotropic thinned virtual array is composed of elements on the aperture boundaries. This is because boundary elements ensures the largest possible array aperture to obtain the largest $Q$, which is in accordance with the observation in [4]. However, as also pointed out by [4], the optimized isotropic thinned virtual array without aperture limit does not exist since the $Q$ value can be arbitrarily large by expanding aperture on every direction so that $\mathrm{CRB}$ can be reduced arbitrarily. This also implies that CRB, which is small enough, can be obtained by a virtual array aperture which is large enough, which is impractical in real-world applications. Besides, the design focused on expanding virtual array aperture renders ambiguity, which is not suitable for the applications which needs enhanced DOA estimation performance.

Meanwhile, the isotropic condition in (30) adds the constraint that the virtual array is symmetric around the gravity center of the aperture, which is difficult to satisfy too. Furthermore, even with constraints on the array aperture, since the array only contains boundary elements, the best possible isotropic array still suffers from high sidelobes, which affects the performance of DOA estimation.

To mitigate the effect of high sidelobes from boundary aperture, the following problem is to be considered:

$$
\begin{array}{ll}
\max _{\mathbf{w}} & \mathbf{w}^{T} \mathbf{x}_{\mathbf{x}} \\
\text { s.t. } & \mathbf{w} \in\{0,1\}^{M_{t} M_{r}} \\
& \mathbf{w}^{T} \mathbf{x}=0 \\
& \mathbf{w}^{T} \mathbf{y}=0 \\
& \mathbf{1}^{T} \mathbf{w}=K \\
& \mathbf{w}^{T} \mathbf{x}_{\mathbf{y}}=0 \\
& \mathbf{w}^{T}\left(\mathbf{x}_{\mathbf{x}}-\mathbf{y}_{\mathbf{y}}\right)=0 \\
& \mathbf{w}^{T} \mathbf{C}_{t, j} \mathbf{w} \leq \delta_{t, j},
\end{array}
$$

where $\mathbf{1}$ denotes all-one vector, $\mathbf{C}_{t, j}=\operatorname{real}\left(\mathbf{v}_{t, j} \mathbf{v}_{t, j}^{H}\right)$ and is the cross-correlation vector between the target steering vector and the steering vector of the $j$ th interference, and $\delta_{t, j}$ represents desired peak sidelobe.
Note that (34) is not convex due to the binary constraint, and this combinatorial problem is to be solved through exhaustive search. To reduce computational complexity from exhaustive search, it is necessary to seek a balance or a trade-off between computational complexity and efficiency through convex approximation to the original problem. In this paper, a sequential convex approximation of (34) is proposed. Specifically, the binary constraint $\mathbf{w} \in\{0,1\}$ is equivalent to the difference between two convex constraints, i.e.,

$$
\begin{aligned}
& A: \mathbf{w} \in[0,1], \\
& B: \mathbf{w}^{T} \mathbf{w}-\mathbf{w}^{T} \mathbf{1}<0,
\end{aligned}
$$

where 1 denotes a vector of all ones.

This means that the binary constraint $\mathbf{w} \in\{0,1\}$ in (34) can be expressed as a maximization problem, namely,

$$
\begin{array}{ll}
\max _{\mathbf{w}} & \mathbf{w}^{T} \mathbf{w}-\mathbf{w}^{T} \mathbf{1} \\
\text { s.t. } & \mathbf{w} \in[0,1] .
\end{array}
$$

It is evident that the objective function does not meet the requirement for the maximization problem. However, the term can be approximated in an affine form by its first-order Taylor decomposition. Note that the derivative of $\mathbf{w}^{T} \mathbf{w}$ is $2 \mathbf{w}$, and thus the objective function in the $k$ th iteration can be approximated by

$$
2 \mathbf{w}^{T} \mathbf{w}^{(k)}-\mathbf{w}^{(k) T} \mathbf{w}^{(k)}-\mathbf{w}^{T} \mathbf{1}
$$

Therefore, (34) can be approximated by iterative convex problems as follows:

$$
\begin{array}{ll}
\max _{\mathbf{w}} & \mathbf{w}^{T}\left(\mathbf{x}_{\mathbf{x}}+2 \mu \mathbf{w}^{(k)}-\mu \mathbf{1}\right)-\mathbf{w}^{(k) T} \mathbf{w}^{(k)} \\
\text { s.t. } & \mathbf{w} \in\{0,1\}^{M_{t} M_{r}} \\
& \mathbf{w}^{T} \mathbf{x}=0 \\
& \mathbf{w}^{T} \mathbf{y}=0 \\
& \mathbf{1}^{T} \mathbf{w}=K \\
& \mathbf{w}^{T} \mathbf{x}_{\mathbf{y}}=0 \\
& \mathbf{w}^{T}\left(\mathbf{x}_{\mathbf{x}}-\mathbf{y}_{\mathbf{y}}\right)=0 \\
& \mathbf{w}^{T} \mathbf{C}_{t, j} \mathbf{w} \leq \delta_{t, j},
\end{array}
$$

where $\mu$ is the parameter controlling CRB and the degree of thinning in the array. By adjusting $\mu$ properly, the trade-off between the CRB and the degree of thinning can be achieved.

The direct thinning of virtual array without priori direction is summarized in Table 1.

3.2. Thinned Virtual Array with Priori Direction. It can be inferred from (17), (19), and (20) that each element in the Fisher information matrix is connected to both the DOA of target and array configuration. Such connection also implies that the array configuration with optimized CRB performance is angle specific, namely, an array that is optimal for one target direction is not for another target direction. 
TABLE 1: Thinning virtual array without priori direction.

\begin{tabular}{lc}
\hline Steps & Procedure \\
\hline Step 1 & Initialize parameters $\mu, \mathbf{w}^{(0)}$, and $I$; set $k=0$ \\
Step 2 & If sidelobe level is to be considered, determine $j$ and $\delta_{t, j}$ \\
Step 3 & Solve problem (32) or (34) to obtain $\mathbf{w}^{(k)}$ \\
Step 4 & $k=k+1$; if $k<I$, go to step 3; if $k \geq I$, go to step 5 \\
Step 5 & $\mathbf{w}^{(I)}$ consists of indices of selected active elements \\
\hline
\end{tabular}

Considering the condition with priori direction, the isotropic virtual array proposed in Section 3.1 is not the most desirable. Instead, the array only needs to detect certain regions of interest with high precision while it still preserves the capacity to detect targets outside those regions. If priori information of target direction is available, e.g., from the results from detection of the isotropic array over the whole angle region, the optimal thinned virtual array can be obtained through optimizing the CRB matrix over the sector of interest, which exploits the DOF from direct thinning of virtual array and the connection between CRB and virtual array configuration.

The elevation $\theta$ and azimuth $\phi$ of a single target is assumed priori known in the following discussions. Since the CRB matrix is the inverse of the Fisher information matrix, the elements on the main diagonal of CRB matrix can be expressed as

$$
\begin{aligned}
& \mathbf{C}_{\theta \theta}=\frac{1}{G} \cdot \frac{1}{\sin ^{2} \theta} \cdot \frac{Q_{w x x} \sin ^{2} \phi+Q_{w y y} \cos ^{2} \phi-Q_{w x y} \sin (2 \phi)}{Q_{w x x} Q_{w y y}-Q_{w x y}^{2}}, \\
& \mathbf{C}_{\phi \phi}=\frac{1}{G} \cdot \frac{1}{\cos ^{2} \theta} \cdot \frac{Q_{w x x} \cos ^{2} \phi+Q_{w y y} \sin ^{2} \phi-Q_{w x y} \sin (2 \phi)}{Q_{w x x} Q_{w y y}-Q_{w x y}^{2}} .
\end{aligned}
$$

The derivation for (39) and (40) are provided in the supplementary material.

To optimize the CRB of virtual array, the trace of the $\mathrm{CRB}$ matrix is the target, which is

$$
\operatorname{tr}(\mathbf{C})=\frac{1}{G} \cdot \frac{\alpha Q_{w x x}+\beta Q_{w y y}+\zeta Q_{w x y}}{Q_{w x x} Q_{w y y}-Q_{w x y}^{2}},
$$

where

$$
\begin{aligned}
& \alpha=\frac{\sin ^{2} \phi}{\sin ^{2} \theta}+\frac{\cos ^{2} \phi}{\cos ^{2} \theta}, \\
& \beta=\frac{\cos ^{2} \phi}{\sin ^{2} \theta}+\frac{\sin ^{2} \phi}{\cos ^{2} \theta}, \\
& \zeta=\frac{\sin (2 \phi)}{\cos ^{2} \theta}-\frac{\sin (2 \phi)}{\sin ^{2} \theta} .
\end{aligned}
$$

The elements in the thinned virtual array is selected by choosing the $M_{t} M_{r} \times 1$ binary vector $\mathbf{w}$, where elements with index of zero values is not included in the thinned virtual array and elements with index of ones is included in the thinned virtual array. The design of thinned virtual array with minimized trace of the CRB matrix can therefore be represented as

$$
\begin{array}{ll}
\min _{\mathbf{w}} & \frac{\mathbf{w}^{T}\left(\widetilde{\alpha} \mathbf{x}_{\mathbf{x}} \mathbf{1}^{T}+\widetilde{\beta} \mathbf{y}_{\mathbf{y}} \mathbf{1}^{T}+\widetilde{\zeta} \mathbf{x}_{\mathbf{y}} \mathbf{1}^{T}\right) \mathbf{w}}{\mathbf{w}^{T}\left(\mathbf{x}_{\mathbf{x}} \mathbf{y}_{\mathbf{y}}^{T}-\mathbf{x}_{\mathbf{y}} \mathbf{x}_{\mathbf{y}}^{T}\right) \mathbf{w}} \\
\text { s.t. } & \mathbf{w}^{T} \mathbf{x}=0 \\
& \mathbf{w}^{T} \mathbf{y}=0 \\
& \mathbf{1}^{T} \mathbf{w}=K,
\end{array}
$$

where $\tilde{\alpha}=(\alpha / K), \widetilde{\beta}=(\beta / K)$, and $\widetilde{\zeta}=(\zeta / K)$.

Note that problem (45) is a fraction of quadratic terms which is nonconvex, thus cannot be solved using convex methods. To avoid large computational complexity from exhaustive search, problem (45) can be approximated by introducing matrix variable $\mathbf{W}$. According to the property of the trace of matrix, (45) can be rewritten as

$$
\begin{array}{ll}
\min _{\mathbf{w}, \mathbf{W}} & \frac{\operatorname{tr}(\mathbf{W N})}{\operatorname{tr}(\mathbf{W D})} \\
\text { s.t. } & \mathbf{w}^{T} \mathbf{x}=0 \\
& \mathbf{w}^{T} \mathbf{y}=0 \\
& \mathbf{1}^{T} \mathbf{w}=K, \\
& \mathbf{W} \geq \mathbf{w w}^{T},
\end{array}
$$

where $\mathbf{N}=\widetilde{\alpha} \mathbf{x}_{\mathbf{x}} \mathbf{1}^{T}+\widetilde{\beta} \mathbf{y}_{\mathbf{y}} \mathbf{1}^{T}+\widetilde{\zeta} \mathbf{x}_{\mathbf{y}} \mathbf{1}^{T}$, and $\mathbf{D}=\mathbf{x}_{\mathbf{x}} \mathbf{y}_{\mathbf{y}}^{T}-\mathbf{x}_{\mathbf{y}} \mathbf{x}_{\mathbf{y}}^{T}$.

Note that the fraction structure $(\operatorname{tr}(\mathbf{W N}) / \operatorname{tr}(\mathbf{W D}))$ is still nonconvex. To facilitate the solution of (46), it should be noted that the fractional problem is usually transformed as the following problem:

$$
F(\eta)=\operatorname{tr}(\mathbf{W N})-\eta \operatorname{tr}(\mathbf{W D})
$$

(47) can be solved using the Dinkelbach method, which is briefly given as follows:

Step 1: initialize the parameter $\eta^{(1)}$ and $\varepsilon$, where $\eta^{(1)}$ is the initial value of $\eta$ and $\varepsilon$ is the threshold value where the function $F(\eta)$ converges.

Step 2: given $\eta^{(k)}$, the following problem is to be solved to obtain the optimal value $\mathbf{w}^{(k)}, \mathbf{W}^{(k)}$ and corresponding function value $F\left(\eta^{(k)}\right)$ :

$$
\begin{array}{ll}
\min _{\mathbf{w}, \mathbf{W}} & F\left(\eta^{(k)}\right) \\
\text { s.t. } & \mathbf{w}^{T} \mathbf{x}=0 \\
& \mathbf{w}^{T} \mathbf{y}=0 \\
& \mathbf{1}^{T} \mathbf{w}=K, \\
& \mathbf{W} \geq \mathbf{w} \mathbf{w}^{T},
\end{array}
$$

Step 3: if $F\left(\eta^{(k)}\right) \leq \varepsilon$, the iteration terminates; $\mathbf{w}^{(k)}$ and $\mathbf{W}^{(k)}$ are the output. Otherwise, let 


$$
\eta^{(k+1)}=\frac{\operatorname{tr}\left(\mathbf{W}^{(k)} \mathbf{N}\right)}{\operatorname{tr}\left(\mathbf{W}^{(k)} D\right)},
$$

and go back to Step 2 .

It should be noted that the optimal parameter $\mathbf{w}^{(k)}$ does not meet the constraint of the original problem (45), and optimal thinned binary vector is to be deduced by solving the optimal integer constrained problem. Specifically, in order to obtain vector $\mathbf{w}$ with zeros and ones as each element, the following problem is to be solved.

$$
\begin{array}{ll}
\min _{\mathbf{w}} & \left\|\mathbf{w}-\mathbf{w}^{(k)}\right\| \\
\text { s.t. } & \mathbf{w} \in\{0,1\}^{M_{t} M_{r}} \\
& \sum_{i=1}^{M_{t} M_{r}} \mathbf{w}_{i}=K .
\end{array}
$$

The design of thinned virtual array with priori direction is summarized in Table 2.

\section{Simulation Results}

In this section, the advantage of the proposed thinned virtual array is validated by simulation results. To give a fair comparison between difference array configurations, numerical simulations in several scenarios are to be considered. First, in the case without priori direction, the virtual array formed by linear transmit array and receive array is thinned, which can be divided into two types, namely, the isotropic (type 1) which only cares about CRB performance and another type (type 2) that takes into consideration both the $\mathrm{CRB}$ and sidelobe performance. The performance of type 1 and type 2 are compared to demonstrate the difference between these two methods. Next, in the case with priori direction, the virtual array formed by square transmit array and receive array is thinned, which involves three types, namely, the isotropic (type 3) which does not consider the priori direction, the directional (type 4) which is thinned based on the priori direction, and the one with wrong priori direction (type 5). The performance of type 3, type 4 , and type 5 are compared to demonstrate that type 4 has the lowest total CRB which achieves better DOA estimation performance. Lastly, to prove the universal efficacy of the proposed thinned virtual array, the total CRB of multiple different directional thinned virtual arrays (type 4), obtained with corresponding elevation and azimuth, is compared with the total CRB of the isotropic (type 3) thinned virtual array and that of the one with priori direction other than the actual target direction (type 5).

4.1. Example 1. To provide comparison between different linear thinned virtual arrays, a linear MIMO radar array is considered, which is composed of $M_{t}=5$ transmit elements and $M_{r}=5$ receive elements. The interelement distance $d_{t}$ is half of wavelength in transmit array, and the distance between elements in the receive array is set as $d_{r}=M_{t} d_{t}$ to expand array aperture as much as possible. In different
TABLE 2: Thinning virtual array with priori direction.

\begin{tabular}{lc}
\hline Steps & Procedure \\
\hline Step 1 & Initialize parameters $\mathbf{W}^{(0)}, \mathbf{w}^{(0)}, \eta^{(0)}, \varepsilon, K$, and $(\theta, \phi)$ \\
Step 2 & Set $k=0$ \\
Step 3 & Obtain parameter $\alpha, \beta$, and $\zeta$ from $(\theta, \phi)$ \\
Step 4 & Given $\eta^{(k)}$, solve problem $(43)$ \\
Step 5 & Obtain $\mathbf{w}^{(k)}$ and $\mathbf{W}^{(k)}$ \\
\hline
\end{tabular}

thinned virtual arrays, the number of virtual elements is chosen to be $K=10$. Assume a single target locates at $\theta=10^{\circ}$, and the target direction is estimated using the maximum likelihood method. For type 2 array, the maximum allowed sidelobe is set to be $\zeta=-10 \mathrm{~dB}$. Problems (33) and (34) are solved separately to obtain the optimal type 1 array and type 2 array, which is shown in Figures 3 and 4.

As shown in Figures 3 and 4, since it only considers the CRB performance, the elements in the optimal type 1 array is concentrated on two sides of the array to achieve the largest aperture which in turn produces the largest moment of inertia. In contrast, the distribution of elements in the optimal type 2 array is more even since it considers counteracting sidelobes.

The overall beampattern of two types of virtual array in conventional beamforming is exhibited in Figure 5.

As revealed in Figure 5, the optimal type 1 array has narrower mainlobe, which agrees with the fact that type 1 array concentrates its elements on the edge of its aperture. However, higher sidelobe also appears in the isotropic type 1 array since virtual elements concentrate on two sides of the virtual aperture. In contrast, the optimal type 2 array has lower sidelobe than type 1 array does, but the mainlobe width of the optimal type 2 array is wider than that of optimal type 1 array due to the changed distribution of elements in the virtual array. The observation above reveals proper balance or trade-off between mainlobe width and sidelobe level, depending on actual applications.

To give a fair comparison between different virtual arrays and minimize the effect of DOA estimation methods, the maximum likelihood method is adopted in this section. Note that the DOA estimates by the maximum likelihood method is also the maximum likelihood estimate of true target directions, which converges to $\mathrm{CRB}$ asymptotically. The Mean Square Error (MSE) and CRB of different thinned virtual array under different SNR conditions are depicted in Figure 6.

As indicated in Figure 6, the CRB of type 1 array is lower than the CRB of type 2 array. Nevertheless, in the low SNR condition ranging from $0 \mathrm{~dB}$ to $5 \mathrm{~dB}$, the threshold SNR of type 2 array is lower than that of type 1 array. This implies that type 2 array performs better in low SNR region than type 1 array. The comparison between two types of thinned virtual array indicates that incorporating the beampattern performance into the design process, lower SNR threshold or better noise reduction capacity can be achieved which helps to improve DOA estimation performance in low SNR region. 


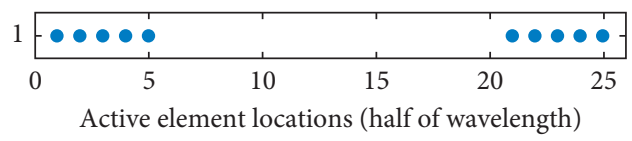

Figure 3: Type 1 array configuration.

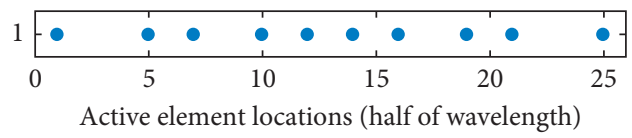

FIgURE 4: Type 2 array configuration.

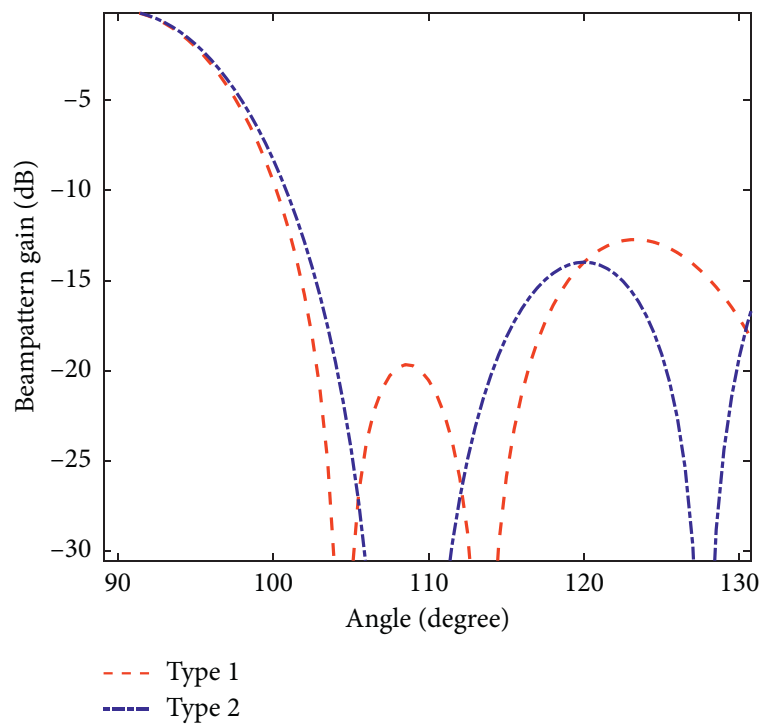

FIgURE 5: Beampattern of type 1 and type 2 arrays.

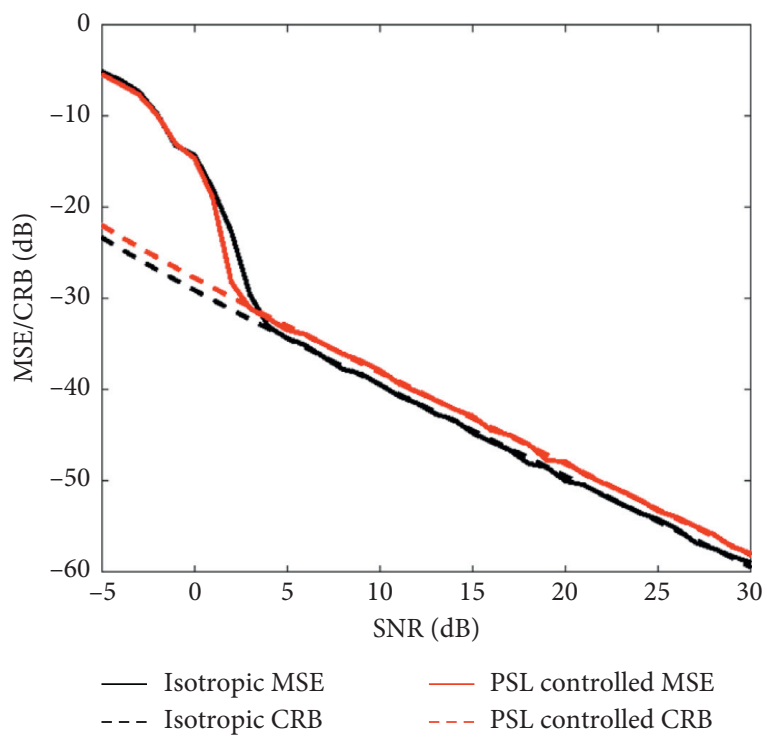

Figure 6: Mean Square Error and Cramer-Rao Bound of type 1 and type 2 array.

4.2. Example 2. In this example, the performance of different thinned virtual arrays is compared in the scenario with priori direction.
Consider a planar MIMO radar array, where $M_{t}=9$ and $M_{r}=9$. Both transmit and receive arrays take on uniform square structure with interelement distance being half of wavelength, as depicted in Figures 7 and 8 . This indicates that the virtual array exhibits a $5 \times 5$ square layout with 25 effective virtual elements, as depicted in Figure 9. Assume that a single target locates with elevation $\theta=18^{\circ}$ and azimuth $\phi=156^{\circ}$. Problem (33) and problem (46) are solved to obtain the isotropic thinned virtual array which does not take into account the priori direction (type 3) and the directional thinned virtual array which incorporates the priori direction in the design of thinned virtual array (type 4 ). Both the two types of thinned virtual array structures are shown in Figures 10 and 11 .

As depicted in Figures 10 and 11, the elements in the optimal type 3 array are located on the boundary of array and are symmetric around the gravity center of virtual array aperture. In contrast, the elements in the optimal type 4 array which takes into account priori direction do not locate on the boundary of array but conforms to distribution that changes according to the priori direction. Even though elements are not on the boundary of the aperture of type 4 array, they are still symmetric around the gravity center due to the symmetry of moment of inertia and the even number of elements.

Similarly to the case in Example 1, the correlation method is exploited to estimation DOA of the target by two types of thinned virtual arrays. In different SNR conditions, the total MSE of both elevation and azimuth and the total $\mathrm{CRB}$ is shown in Figure 12.

As exhibited in Figure 12, the total CRB of the optimal type 4 array is lower than that of the optimal type 3 array. The comparison between the total MSE of two types of array indicates that the total MSE of the optimal type 4 array is close to CRB even in low SNR condition (less than $-2 \mathrm{~dB}$ ); the total MSE of the optimal type 3 array, however, does not converge to the total CRB until the SNR is larger than $3 \mathrm{~dB}$. In other words, the SNR threshold of type 4 array is $5 \mathrm{~dB}$ lower than that of type 3 array. These observations imply that the priori direction information contributes to total MSE performance in the low SNR condition. The optimal type 4 array introduces priori information and enjoys low total MSE even in low SNR condition, while the optimal type 3 array has higher SNR threshold since the priori direction information is not present in the isotropic thinned virtual array.

4.3. Example 3. In this example, the generality of the performance of directional thinned virtual array in cases with different priori directions is demonstrated. Assume that the SNR is $10 \mathrm{~dB}$, the azimuth angles changes from $0^{\circ}$ to $180^{\circ}$ with $1^{\circ}$ as step; other conditions remain the same as in Example 2. In each case with different azimuth angles, the total CRB of isotropic thinned virtual array (type 3), directional thinned virtual array (type 4), and the thinned virtual array with priori azimuth at 150 (type 5) are depicted in Figure 13. 


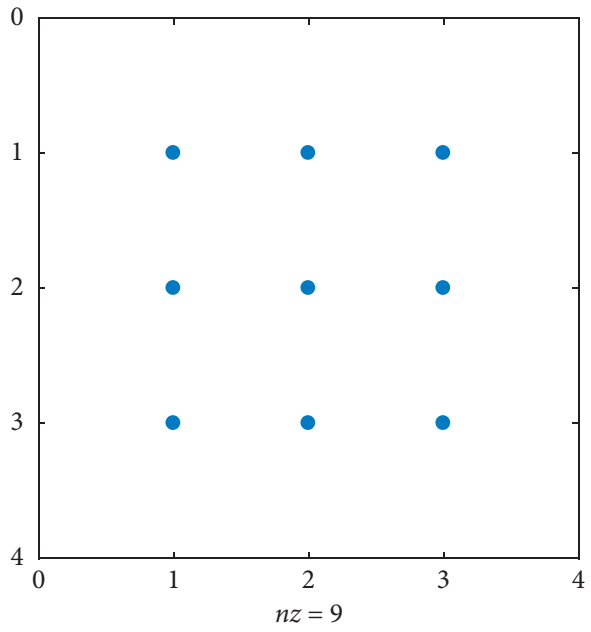

FIgURE 7: Transmit array configuration.

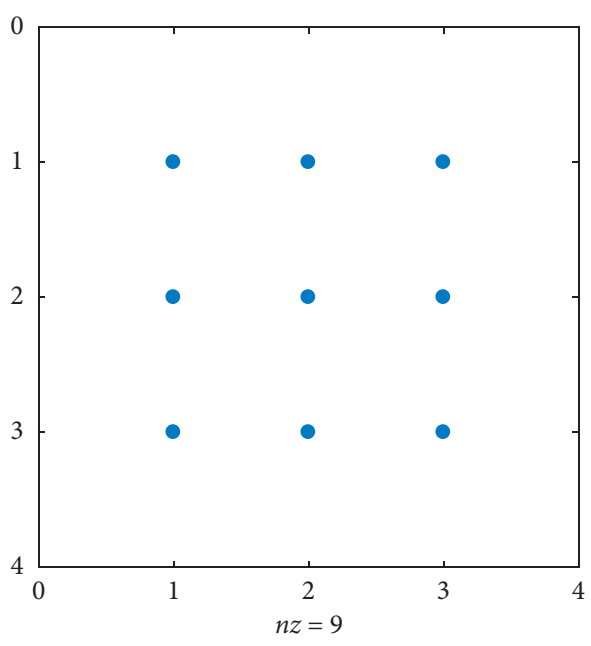

Figure 8: Receive array configuration.

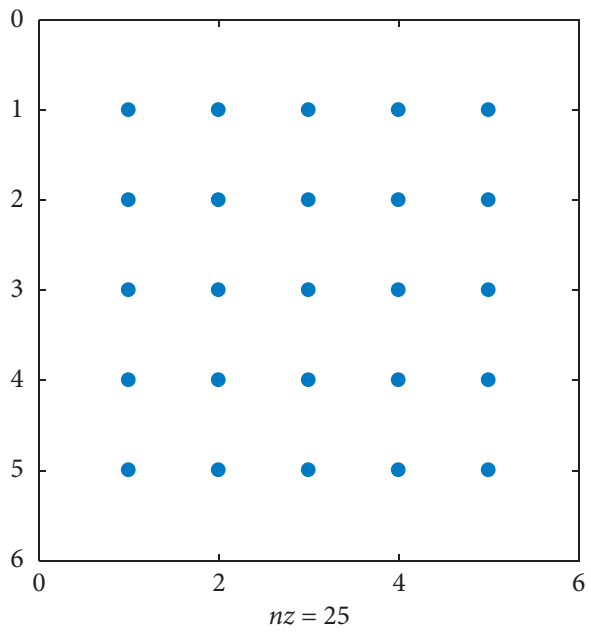

FIGURE 9: Full virtual array configuration.

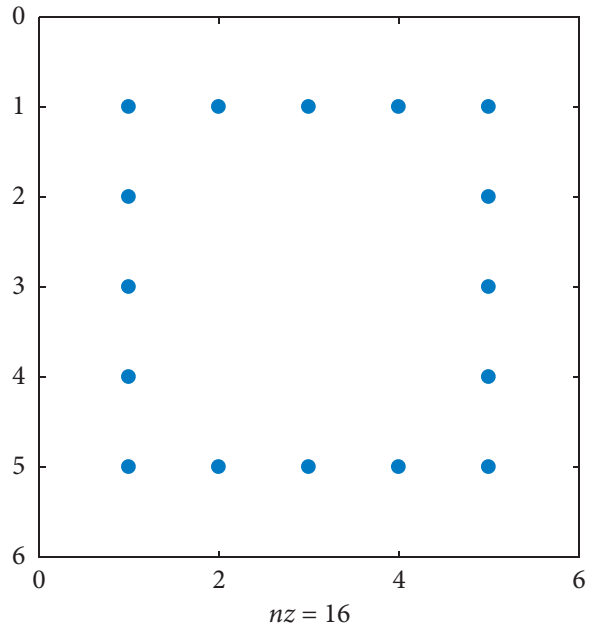

FIgURE 10: Type 3 thinned virtual array configuration.

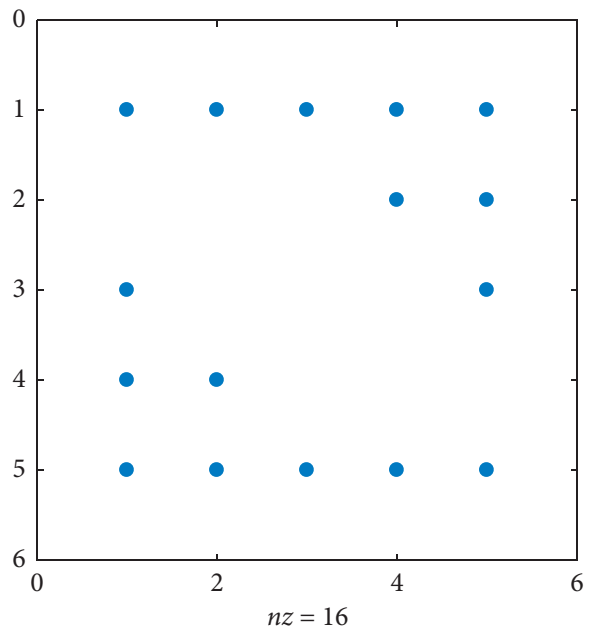

Figure 11: Type 4 thinned virtual array configuration.

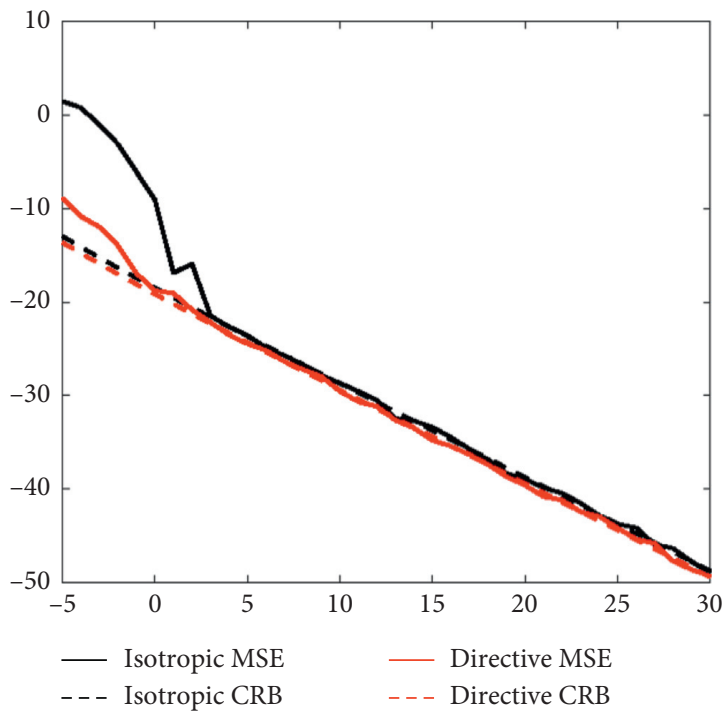

FIgURE 12: Total MSE and total CRB of type 3 and type 4 array. 


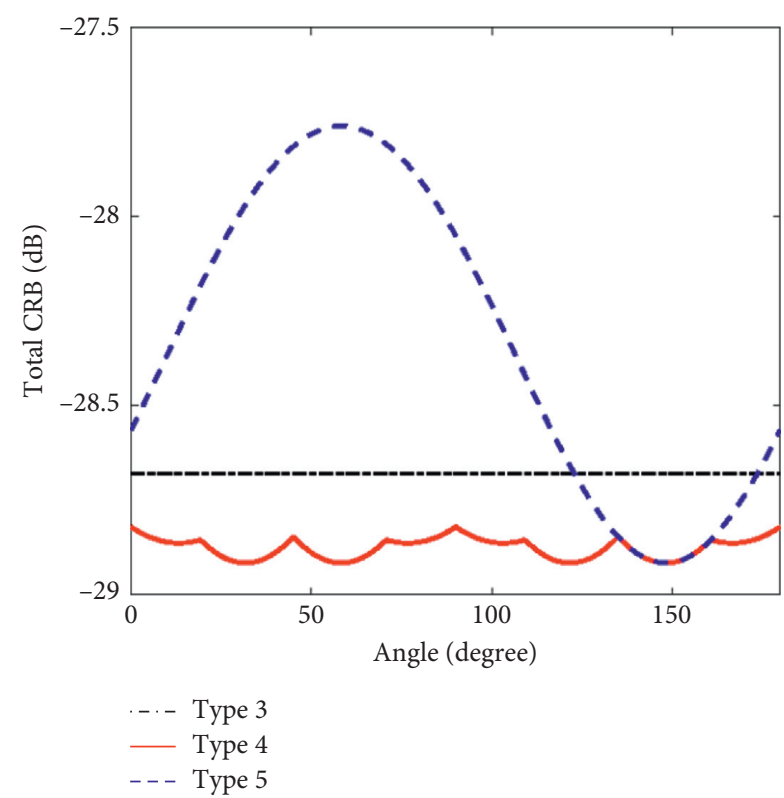

FIGURE 13: Total CRB of different virtual arrays against different azimuth with fixed elevation.

As displayed in Figure 13, the total CRB of the optimal type 3 array is constant over different azimuth angles since the virtual array is isotropic. In comparison, the total CRB of the optimal type 4 array experiences fluctuation over different azimuth angles, but remains close and is lower than that of isotropic type 3 array. For the directional type 5 array, its total CRB is low when the direction of actual target coincides with the priori one; however, when the direction of the actual target is different from the priori direction, the total CRB increases, indicating the direction-finding performance of virtual thinned array is reduced since its DOA estimation is misled by the wrong priori information. These observations indicate that when elevation is fixed yet azimuth sweeps over different angles, by taking the optimal thinned virtual array on each direction, the total CRB remains lower than that of isotropic thinned virtual array while remaining constant compared to the optimal directional thinned virtual array with fixed priori direction. These observations also indicate that reconfiguration of thinned virtual array according to priori information of spatial targets is a feasible way of obtaining total CRB which is lower than that of isotropic thinned virtual array over different spatial angles.

\section{Conclusions}

In this paper, the method for designing thinned virtual arrays in the MIMO radar is proposed. The connection between total CRB and virtual array is analysed, based on which the thinning of virtual array is proposed both in case with and without priori directions. In order to solve the quadratic fractional problems efficiently, the original nonconvex problem is approximated by a series of convex problems which can be solved iteratively, the solution of which is used as the reference point in the optimal integer constraint problem to obtain the binary solution which satisfies the requirement of the original problem. Simulation results show that the thinned virtual array can achieve proper balance between DOA estimation and sidelobe performance when no priori direction is present. When priori direction is available, the directional thinned virtual array achieves lower total CRB and total MSE than the isotropic thinned virtual array without priori direction does. Future work may include implementation of rapid changing of virtual array structure through matched filters and adaptive virtual array reconfiguration in real-time applications.

\section{Data Availability}

The data used in the simulation of the manuscript is available in open-source Octave software.

\section{Conflicts of Interest}

The authors declare that there are no conflicts of interest regarding the publication of this paper.

\section{Acknowledgments}

This work was supported in part by the National Natural Science Foundation of China under Grant 61671465.

\section{Supplementary Materials}

Appendix: derivation of elements in the Fisher information matrix and CRB matrix. (Supplementary Materials)

\section{References}

[1] J. Li, X. Zhang, W. Chen, and T. Hu, "Reduced-dimensional ESPRIT for direction finding in monostatic MIMO radar with double parallel uniform linear arrays," Wireless Personal Communications, vol. 77, no. 1, pp. 1-19, 2014.

[2] N. H. Lehmann, E. Fishler, A. M. Haimovich et al., "Evaluation of transmit diversity in MIMO-radar direction finding," IEEE Transactions on Signal Processing, vol. 55, no. 5, pp. 2215-2225, 2007.

[3] H. Chen, Y. Peng, W. Li, and J. Yang, "Manifold studies on fundamental limits of direction-finding multiple-input multiple-output radar systems," IET Radar, Sonar \& Navigation, vol. 6, no. 8, pp. 708-718, 2012.

[4] H. Chen, Z. Zhuang, X. Li, and H. Wang, "Performance bounds of direction finding and its applications for multipleinput multiple-output radar," IET Radar, Sonar \& Navigation, vol. 8, no. 3, pp. 251-263, 2014.

[5] R. D. Fry, D. A. Gray, and L. A. Balzan, "MIMO arrays and array shading," in Proceedings of the 2009 IET International Radar Conference, Guilin, China, 2009.

[6] W. Wang, H. Shao, and J. Cai, "MIMO antenna array design with polynomial factorization," International Journal of Antennas and Propagation, vol. 2013, Article ID 358413, 9 pages, 2013.

[7] S. Gu, K. Li, and X. Ren, "Antenna array design in MIMO radar using NSK polynomial factorization algorithm," International Journal of Antennas and Propagation, vol. 2016, Article ID 4580479, 10 pages, 2016. 
[8] H. Liu, Q. Xu, and G. Jin, "MIMO antenna polynomial weighted average design method of downward-looking array SAR," International Journal of Antennas and Propagation, vol. 2017, Article ID 3029847, 18 pages, 2017.

[9] J. Dong, Q. Li, and W. Guo, "A combinatorial method for antenna array design in minimum redundancy MIMO radars," IEEE Antennas and Wireless Propagation Letters, vol. 8, pp. 1150-1153, 2009.

[10] J. Dong, R. Shi, W. Lei, and Y. Guo, "Minimum redundancy MIMO array synthesis by means of cyclic difference sets," International Journal of Antennas and Propagation, vol. 2013, Article ID 323521, 9 pages, 2013.

[11] J. Dong, F. Liu, Y. Guo et al., "MIMO radar array thinning optimization exploiting almost difference sets," Optik, vol. 127, no. 10, pp. 4455-4460, 2016.

[12] G. Oliveri and A. Massa, "Genetic algorithm (GA)-enhanced almost difference set (ADS)-based approach for array thinning," IET Microwaves, Antennas \& Propagation, vol. 5, no. 3, pp. 305-315, 2011.

[13] J. Dong, J. Yang, W. Lei et al., “Antenna array design in MIMO radar using cyclic difference sets and simulated annealing," in Proceedings of the 2012 International Conference on Microwave and Millimeter Wave Technology (ICMMT), pp. 1-4, Shenzhen, China, 2012.

[14] Q. Wu, F. Sun, P. Lan, G. Ding, and X. Zhang, "Two-dimensional direction-of-arrival estimation for co-prime planar arrays: a partial spectral search approach," IEEE Sensors Journal, vol. 16, no. 14, pp. 5660-5670, 2016.

[15] S. Qin, Y. D. Zhang, and M. G. Amin, "DOA estimation of mixed coherent and uncorrelated targets exploiting coprime MIMO radar," Digital Signal Processing, vol. 61, pp. 26-34, 2017.

[16] J. Li and D. Jiang, "Low-complexity propagator based two dimensional angle estimation for coprime MIMO radar," IEEE Access, vol. 6, pp. 13931-13938, 2018.

[17] M. Yang, L. Sun, X. Yuan, and B. Chen, "Improved nested array with hole-free DCA and more degrees of freedom," Electronics Letters, vol. 52, no. 25, pp. 2068-2070, 2016.

[18] M. Yang, L. Sun, X. Yuan, and B. Chen, "A new nested MIMO array with increased degrees of freedom and hole-free difference coarray," IEEE Signal Processing Letters, vol. 25, no. 1, pp. 40-44, 2018.

[19] W. Zheng, X. Zhang, and J. Shi, "Sparse extension array geometry for DOA estimation with nested MIMO radar," IEEE Access, vol. 5, pp. 9580-9586, 2017.

[20] J. Shi, G. Hu, X. Zhang, F. Sun, and H. Zhou, "Sparsity-based two-dimensional DOA estimation for coprime array: from sum-difference coarray viewpoint," IEEE Transactions on Signal Processing, vol. 65, no. 21, pp. 5591-5604, 2017.

[21] J. Shi, G. Hu, X. Zhang, and F. Sun, "Sparsity-based DOA estimation of coherent and uncorrelated targets with flexible MIMO radar," IEEE Transactions on Vehicular Technology, vol. 68, no. 6, pp. 5835-5848, 2019.

[22] D. M. Kitavi, K. T. Wong, and C.-C. Hung, "An L-shaped array with nonorthogonal axes-its Cramér-Rao bound for direction finding," IEEE Transactions on Aerospace and Electronic Systems, vol. 54, no. 1, pp. 486-492, 2018.

[23] Y.-h. Tang, X.-f. Ma, W.-x. Sheng, and Y. Han, "Transmit beamforming for DOA estimation based on Cramer-Rao bound optimization in subarray MIMO radar," Signal Processing, vol. 101, pp. 42-51, 2014. 